In the Special Issue on Multicultural Social Justice Leadership Development Guest Editor: Carlos P. Zalaquett, University of South Florida

\title{
Neuroscience and Counseling: Central Issue for Social Justice Leaders
}

\author{
Allen E. Ivey \\ Carlos P. Zalaquett \\ University of South Florida
}

\begin{abstract}
This article summarizes a presentation focusing on basics of neuroscience with special attention to implications for social justice. Participants' questions and suggestions for action are presented.

Keywords. neuroscience, advocacy, social justice, neurogenesis, neuroplasticity, leadership
\end{abstract}

Experiences, thoughts, actions, and emotions actually change the structure of our brains. . . . Indeed, once we understand how the brain develops, we can train our brains for health, vibrancy, and longevity. - John Ratey

Brain research has reached a state of precision where it now has immediate meaning for counseling process and outcome. Neuroscience, neuroimaging, and other neurobiological disciplines have found that measurable structural changes occur in client brains as a result of cognitive and interpersonal therapy. The discovery of neurogenesis-the capacity of the brain to produce new neurons, advanced our 
knowledge of brain development and complemented known information regarding its functional changes (e.g., establishing new neuronal connections or strengthening existing connections). Advances in positron emission tomography (PET) scans and functional magnetic resonance imaging (fMRI) have made it possible to measure these changes in areas of the brain that "light up" or "fire" under various stimulus conditions. And, it is not just the client who develops new neurons and neural nets in the process of counseling; the counselor's brain is changing as well.

Current research in neuroscience and cognitive science is highly supportive of our emphasis on listening, empathic understanding, and building strengths and wellness; and provides ample support for the microskills (Ivey, Ivey, \& Zalaquett, 2010). Somewhat surprisingly, neuroscience's findings on the brain result in a more complete awareness of how environment and culture shape the individual. The neuroscientific evidence demonstrates that we are a unique social species with highly developed cultures and social institutions (Gazzaniga, 2008). According to Caccioppo, Berntson, and Deceity (2010), "Our biology has helped shape the social environments we have created, and our social environment has helped shape our genes, brains, and bodies ( $p$. 676). The bridge between biological and psychological processes is erasing the old distinction between mind and body, between mind and brain-the mind is the brain.

\section{How and why is neuroscience and cognitive science relevant to counseling practice?}

First, neuroscience provides research that suggests most of counseling's theory and practice is on target. It also provides a clearer understanding of why what we do works. Moreover, it provides ideas for improving our work with clients. And, surprisingly, demonstrates that our wellness, social justice, and environmentally based orientation is correct.

Neuroscientific evidence suggests that without a meaningful and effective environment, we cannot grow and change. In counseling, this means that our key word relationship is all that more important and that we need to honor and respect what we have done and what we can do in the future. Counseling builds new brain networks.

The following section illustrates the usefulness of neuroscience for counseling. Neuroscience research also demonstrates the factors that negatively affect the brain.

\section{Basic Neuroscience's Concept Usefulness}

Note that all of the following aspects of brain development and functioning are harmed by stress and trauma, including the many aspects of oppression as defined by social justice advocates.

Neuroplasticity. The brain can change-it is not fixed and it responds to external environmental events and to actions initiated by the individual. The traditional assumption that the brain does not change is wrong. Throughout the lifespan new connections and new neural networks are developed. Regardless of age, genetic 
background, or life experience, change can happen. Schwartz and Begley (2003) note that "Neuroplasticity can result in the wholesale remodeling of neural networks . . . a brain can rewire itself" (p. 13). Effective counseling not only changes minds, it changes brains as well. And this includes our own brain, as helpers.

Neurogenesis. Counseling can support the building of new neurons! One of the most startling findings is that completely new neurons can be generated in the learning process, even in older adults. The idea that we only go "downhill" in later life has been proven wrong and there are many areas of the brain where neurogenesis occurs. We develop new neural networks throughout the lifespan in response to new situations or experiences in the environment. Exercise is particularly important as a lifetime process to ensure brain and physical health. Exercise increases blood flow and the release of positive neurotransmitters such as serotonin. Exercise needs to be part of your treatment regime. Serotonin release is particularly helpful in mitigating depression. If you are sad-walk or run! If you can't run, meditate and use relaxation training. Not only do your clients feel better, their brains are expanding.

The Importance of Attention and Focus. Our basic concepts of attending behavior and attention are measurable through brain imagining and, of course, are required for the learning process that is counseling. When client and counselor attend to the story, the brain of both participants become involved. Factors in attention are arousal and focus. Arousal involves the reticular activating system, at the brain's core, which transmits stimuli to the cortex and activates neurons firing throughout many areas. The executive frontal cortex can determine the direction of attention. Central to this process is the microskill of attending behavior. If you attend with energy and interest, and this is communicated effectively, expect your client to see you as a positive resource. An excellent treatment to facilitate client focus and attention is meditation. Benson and Proctor (2010) suggest that relaxation and meditation helps you "switch off" or "switch on" gene activity associated with health and disease (p. xii).

Clarifying our Understanding of Emotions. Brain imagining reveals that specific emotions fire different parts of the brain. Again, we see that our basic concepts are verified. The amygdala is the major seat of the negative emotions of sad, mad, and fear, but it is also an energizer for learning and absorbing new input and memories. Many areas of the brain are activated by positive emotions. The prefrontal cortex and the hippocampus are obviously important, but the nucleus accumbens sends out signals to the dorsal cinguate cortex and prefrontal cortex, making it possible to focus on the positive. Thinking and feeling positively is heavily influenced by executive cognition functions. 
Focusing on Wellness and the Positives. Counselors focus on negative issues and problems builds a self-reinforcing circularity between the "demons" of the amygdala and the frontal cortex. Result-negative thinking, accompanied by negative feeling, characteristic of depression. Pessimism feeds on itself. However, there is clear research evidence that an effective executive frontal cortex focusing on positives and strengths can overcome the negative. Appropriate medication (e.g. Lexapro, Wellbutrin) can enhance positive thinking through increasing the supply of serotonin. Ellis and Beck's cognitive behavioral counseling does the same thing. "Accentuate the Positive . . . Eliminate the Negative," as in the old popular song. Keep in mind that wellness activities such as exercise, positive reframing of old stories, interpersonal relationships, meditation, and leisure all facilitate our ability to control and "demons" of negative thinking and feeling.

\section{Empathy and Mirror Neurons.}

Empathy is not just an abstract idea; empathy is identifiable and measurable in the physical brain. Research on brain activity validates what the helping field has been saying for years. Decety and Jackson (2004) comment "The basic building blocks [of empathy] are hardwired into the brain and await development through interaction with others. . . . empathy [is] an intentional capacity" (pp. 71, 93).

Let us "unpack" the meaning of that complex sentence and its implications. Mirror neurons are neurons that fire when we behave, think, or feel and they also fire when we see others behave, think, or feel. Mirror neurons enable you to sense and understand what the client is saying and feeling. These neurons fire and even impact your internal bodily responses when you are truly empathically experiencing the world of the client. This is a natural talent that you can encourage and develop by increasing your awareness of the client and noting what happens inside your own body.

And you are awakening the mirror neurons in the client and facilitating their development of new connections in thoughts, feelings, and action. This awakening shows in verbal behavior of clients and the action that they take as a result of the interview. And, as the clients restory their issues, new neural connections are born. Your empathic behavior and the relationship are central to change. Needless to say, this emphasizes the importance of a positive approach to change. If we listen and selectively attend only to problems, this reinforces negative patterns in the brain and will make the change process slow and clumsy.

What we learn here is that the empathic person's brain responds to another person's experience, even though he or she does not actually experience that person's world. Many studies over the years back up this central point. For example, children around their second year indicate concern for others cognitively, emotionally, and behaviorally by comprehending others' difficulties and trying to help. You also may have seen two young children playing together. One falls and starts crying. Even though the second child has not been hurt, he or she also cries. This ability to observe the feelings of others could be considered the developmental roots of empathic understanding. 
Decety points out that the antisocial, criminal personality to has reduced ability to appreciate the emotions of others. There is less firing of mirror neurons in the prefrontal cortex, and this deficit also appears to be a dysfunction of the energizing amygdala and hippocampus (long-term memory). His breakthrough work with children diagnosed with conduct disorder again reveals less activity in mirror neuron areas of the brain. Another important finding is presented by Susan Fiske (2007). She investigated automatic prejudicial responses to the poor using MRI technology. She discovered that these responses go beyond prejudice as many individuals do not see the poor as humans. Research participants shown the photograph of a homeless man demonstrated brains' activation patterns in areas that are "reliably implicated in disgust toward nonhuman objects such as garbage, mutilation, and human waste" (p. 157). Areas consistently associated with the perception of human beings failed to light up, "as if people had stumbled on a pile of garbage" (p. 157). It is possible that training in empathy and the microskills may increase the effectiveness of our mirror neurons and diminish responses such as the one described by Deceity and Fiske.

Also, the nucleus accumbens is related to the "high" from certain recreational drugs. It is particularly responsive to marijuana, alcohol, and related chemicals and thus is key in addiction. When we seek to help an addicted client, we are working against some very powerful parts of the brain. One of our great challenges is helping these clients examine and rewrite their stories and find new actions through healthy alternative highs to replace the strengths of addiction. When you find these clients developing new life satisfactions and interests (wellness), you are influencing them toward behavior that can result in new positive responses in the nucleus accumbens and other parts of their brain. William Glasser, the founder of reality therapy, long ago stated the importance of building positive addictions to combat drugs, antisocial behavior, and alcohol.

\section{How and why is neuroscience relevant to multicultural and social justice counseling?}

Key social justice issues such as poverty, abuse, bullying, racism, sexism, heterosexism, and discrimination against the persons with disabilities, and other forms of oppression are all stressful. Stressful events can curtail normal brain development in newborns and youngsters and negatively affect brain neurogenesis and plasticity in adults and older adults. Thus, these social justice issues are detrimental to the psychological, physical, and social well-being of individuals. Poverty provides a clear illustration of the detrimental effects of unjust conditions.

The World Health Organization, the World Bank, the American Counseling Association, and the American Psychological Association (2000) have all documented the impact of environmental and social conditions on those who are poor. Their conclusion is that poverty is detrimental to the well being of societies and individuals. The greater the income gap between the poorest and the wealthiest in a society, the higher the death rates for infants and adults and the lower the life expectancy for ALL members of that society, regardless of socio-economic status.

Poverty is associated with substandard housing, homelessness, inadequate childcare, unsafe neighborhoods, and under resourced schools. In addition, negative 
environmental factors such as pollutants and contaminants, and lack of potable water have detrimental effects on mental and physical development that perpetuate and contribute to poverty.

Thus, those living in poverty are over 3 times as likely to be uninsured. Lack of affordable health insurance, including mental health and substance abuse coverage, impedes health and well-being. Men and women have higher mortality rates and present higher incidence of osteoarthritis, hypertension, cervical cancer, coronary heart disease, AIDS/HIV infection, and other chronic health conditions. As a consequence those who are poor are sicker and more likely to have disabilities than their nonpoor counterparts, limiting their employment options, straining their financial resources, and increasing their stress levels.

The impact of poverty on young children is also significant and long lasting. Children who are poor are at greater risk than higher income children for a range of problems, including poor academic achievement, poor socioemotional functioning, developmental delays, behavioral problems, asthma, poor nutrition, low birth weight, and pneumonia.

Finally, low-income groups are the targets of discrimination based on their socioeconomic status as well as other social indicators such as gender. Those from middle and upper socioeconomic levels display attitudes and stereotypes that attribute poverty to personal failings rather than to socioeconomic structures and systems; and tend to ignore the strengths and competencies of those from low socioeconomic levels. Public policy and anti-poverty programs also reflect these stereotypes. Counselors, psychologists, and other professionals also present such biases.

In addition, low-income individuals are 2 to 5 times more likely to suffer from a diagnosable mental disorder than those of the highest socioeconomic levels, and poverty poses a significant obstacle to getting help for these mental health problems. Poverty is detrimental to the physical, psychological, and social health of individuals.

\section{What impact does stress have on the brain?}

Stressful experiences disengage the frontal lobes, which, over time can lead to:

- Impulsivity

- Short-sightedness

- Aggressive behavior

- Increased anxiety

- Depression

- Alcohol and drug abuse

- Learning disorders

- Stress-related diseases

The subcortical arousal system bypasses the frontal lobe's executive functioning to prompt our stress response: 
- Increased heart rate

- Increased respiratory rate

- Strengthened muscle tone

Stressful experiences lead to dysfunctions of the prefrontal cortex, including critical areas regulating judgment, planning, decision-making, moral reasoning, and sense of self. Out of this comes a wide variety of so-called disorders, which are really a result of an often insane, oppressive environment. Discrimination shows in both the mind and body.

We understand that stress is necessary for learning. We need mental and physical challenges to grow. So do not aim for a totally neutral, peaceful environment, except as a rest period (for yourself and your clients). During stress, glucocorticoids released which provide short-term pleasure; but, long-term continued stress results in damage to the brain and loss of brain plasticity. During stressful times, excessive cortisol is released in to the brain that impact our attention-and, if a sufficient amount is released in trauma or extended stress, long-term toxicity results, with the loss of neural connections such as post-traumatic stress.

\section{Our responsibility does not end with individual therapy}

Oppression brings us many clients-war, rape, abuse, poverty, racism, sexism-all forms of discrimination. The family, groups, community, and nation can benefit from our knowledge. We have a responsibility to inform clients about how oppression impacts their problems and we need to act in society to promote change. We also need to influence our governing and regulation bodies.

\section{CACREP and the Brain}

Getting our field to accept and learn this new area will be challenging. We know of no curriculum that includes a serious discussion of how we can use neuroscience and cognitive science in counseling and therapy practice. Fortunately, the new training and educational standards of the major accrediting association in the counseling field (CACREP) anticipated this future in the 2009 standards. On page 9 we find the following statement, incorporating these new ideas:

HUMAN GROWTH AND DEVELOPMENT—studies that provide an understanding of the nature and needs of persons at all developmental levels and in multicultural contexts, including all of the following:

a. theories of individual and family development and transitions across the life span;

b. theories of learning and personality development, including current understandings about neurobiological behavior . . . (italics added)

The CACREP standards' statement provides a rational and direction for our new future. And we are now at a point where neuroscience and its related areas can and will provide a powerful impetus for adding new content to our curriculum and practice. Unless we become aware of this new paradigm and change, we are in danger of falling behind in 
our daily practice, teaching, and research. Cacioppo and Jean Decety have written "psychological science in the 21st century can and should become not only the science of overt behavior, not only the science of the mind, but the science of brain function." They point out that major changes in our curriculum and practice will be necessary in this new world. Not only does neuroscience clearly indicate that we are on the right track, but it also demonstrates the need for updating our field, if we are to remain current and relevant.

Many worry about the "medical model," and a possible focus on pathology. However, you will find that neuroscientists have a strong environmental orientation-client development over the lifespan clearly impacts the brain. Evidence is that effective counseling and therapy can change the brain in positive ways. In truth, neuroscience reinforces counseling's wellness model!

\section{Looking to the Future}

Likhtik, Popa, Apergis-Schoute, Fidacaro, and Paré (2008) present a particularly interesting and relevant piece of research. They have identified specific neurons in the amygdala that affect PTSD, anxiety, and depression. Using classic Pavlovian fear conditioning methods, they were able to change these neurons through presenting the positive image, which overcame the negative.

We can cite several other studies supporting the fact that counseling builds new neurons and neural networks to overcome stress, anxiety, and fear. Positive psychology and the wellness approach are likely to be as effective or more effective than our traditional approach to helping. But, we cannot be all positive for we need to remain aware of social conditions. Even our genes and DNA are impacted by trauma and oppression. On the other hand, evidence such as the above suggested that interventions can still make a positive difference.

Neuroscience research provides an important biological foundation for understanding the impact of our work. The very act of interviewing and counseling produces changes in client memory (and your own). Always be aware that new ideas and learning are being constructed in the session. We suggest that you continue to study and learn about brain structures and functions as new findings may provide further support for our work and suggest specific guidelines for practice.

Data are beginning to suggest that effective counseling in many cases can be more long lasting than medication. Why? We are clearly impacting neurotransmitters in the process of developing new neural networks that contain our thoughts and feelings, leading to behaviors. And, we are simultaneously teaching skills that will last after medication has ceased.

Brain research is not in opposition to the cognitive, emotional, behavioral, and meaning emphasis of interviewing and counseling. Rather, it will help us pinpoint types of interventions that are most helpful to the client. In fact, one of the clearest findings is that the brain needs environmental stimulation to grow and develop. You can offer a healthy atmosphere for client growth and development. We advocate the integration of 
counseling, psychotherapy, neuroscience, molecular biology, and neuroimaging, and the infusion of knowledge from such integrated fields of study to practice, training, and research.

\section{Responses to Comments and Feedback from Academy participants}

Audience response was positive to the presentation. Here are key questions and comments, with Allen's responses.

The relevance of neuroscience. Allen was asked to speak about the relevance of neuroscience and its relevance for both counseling and social justice action. He began his response to this topic with the one item-exercise-that research has shown most important for brain health and, simultaneously, physical health as well. He pointed out that if people only remember one thing from today's presentation, this is what they should recall.

Exercise will:

- Increase immune function, strengthen bones, lower cholesterol

- $\quad$ Reduce sick days $25 \%$ to $50 \%$

- Reduce Alzheimer's risk 40\%

- Reduce High blood pressure $50 \%$

- Reduce cancer risk 30\% (Recurrence reduced 50\%)

- Reduce colon cancer $60 \%$

- Reduce stroke $27 \%$

- As effective as medications and therapy for depression!

- Read John Ratey's SPARK!

This obviously speaks to all clients, but is something that we too often forget when we work with social justice issues. Those who are poor, depressed, suffer from posttraumatic stress will all benefit from exercise as a part of your standard treatment procedures.

Beyond that social justice action suggests that we need to engage in education, develop community projects that work toward health, and influence social policies and procedures that maintain injustice and inequalities.

CACREP and our future. Needless to say neuroscience and the ideas expressed in this presentation are not yet prominent in the literature, but CACREP has anticipated this future. Here is what they say on Page 9 of the 2009 standards:

- HUMAN GROWTH AND DEVELOPMENT...

- b. theories of learning and personality development, including current understandings about neurobiological behavior . . . (italics added)

Given this, it is clear that counseling curricula are going to change, but it is important that these changes include critical information on social justice. One of the most important arguments for integrating social justice into our curricula and practice is the 
fact the social injustice is so much a part of many of our client's experience. As presented above, neuroscience and brain findings provide clear evidence that oppression impacts our clients negatively in terms of physical and mental health. Let us use this new information to work more effectively for social change. Social justice is our professional obligation.

Provide an overview of how the brain works immediately in the presentation, not in the middle. Sequencing is always an issue in any presentation. At times, we have presented brain basics first, then moved to social justice implication.

How do we apply information learned? The most important application is to become aware that counseling and psychology have become limited in their awareness and are currently far behind the curve of psychiatry and medicine. Each of us has the responsibility to take time daily to get in touch with a challenging, complex, and difficult area.

We need professors to start teaching courses in this area. Fortunately, this has started. Allen gave the first presentation on neuroscience and counseling at the American Counseling Association in Honolulu two years ago. At that point, nothing was happening, but now many people are becoming involved and interested.

Here is how I use it in therapy right now.

1. I inform clients as to how the brain reacts to stress and that stress is part of virtually every client issue. I tell them how stress management and successful therapy will build new neurons and neural networks.

2. I stress that action and doing something beyond the interview is vital for learning and change. (We give insufficient attention to this issue in our theories and practice courses. Relapse prevention or some variation for systemic generalization from the session is essential.)

3. I use my understanding of emotions and can use reflection of feeling more precisely. Along with that, I am aware that positive stories and events can counteract the damage that the client brings us. Too many counselors spend time on problems, thus reinforcing negative emotions deep in the brain. This has to end.

4. I now prescribe exercise to every client. This increases blood flow to the brain and, of course, is part of body health.

5. I inform clients how trauma and oppression and racism can impact the brain negatively. This leads to an emphasis on cultural pride, wellness, and examination of ways to deal with both personal and institutional racism.

6. I could go on, but virtually all community social justice action are part of effective treatment. It is time to get out of our office!

Do you have case examples to help make information practical and useful for educators? I do, but this case information comes from my own video or live demonstrations. I like to show DVD's illustrating my interviewing style. If I do a live demonstration, I stop periodically in the middle of the session and discuss example neuroscience issues in the here and now. My knowledge in this area now guides my practice and teaching. 
This type of knowledge and expertise needs to be part of all our practice and teaching.

Can you provide some useful research and thoughtful sources information on this topic? Here are just a few possibilities for further examination and study.

Decety, J., \& Jackson, P. (2004). The functional architecture of human empathy. Behavioral and Cognitive Neuroscience Reviews, Volume 3, Number 271-100. Retrieved from (http://home.uchicago.edu/\%7Edecety/publications/Decety CDPS06.pdf.

The best article we have read on empathy and it shows neuroscience implications as well.

Grawe, K. (2007). Neuropsychotherapy: How the neurosciences inform effective psychotherapy. Mahway, NJ: Erlbaum. Grawe, sadly, is deceased, but read his obituary at $h t t p: / / w w w . p s y c h o t h e r a p y r e s e a r c h . o r g / d i s p l a y c o m m o n . c f m ? a n=1 \&$ subarticlenbr=50.

This is the book that best provides specifics of connections between neuroscience and therapy.

Ivey, A. (2009). Neuroscience and counseling: Implications for microskills and practice (Video). Framingham, MA: Microtraining Associates. (www.emicrotraining.com)

Allen discusses his integration of neuroscience and counseling with a focus on microskills.

Ivey A., Ivey, M., \& Zalaquett, C. (2010) Intentional interviewing and counseling: Facilitating client development in a multicultural world ( $7^{\text {th }} \mathrm{Ed}$.), Belmont, CA: Brooks/Cole/Cengage.

Appendix II details many specifics of the brain and key functions. You'll also encounter how we impact neurotransmitters. The book has many specifics on practical applications.

Ratey, J. (2001) A user's guide to the brain. New York: Vintage.

(http://en.wikipedia.org/wiki/John_Ratey)

This is the ideal follow-up or substitute for Sapolosky's lectures. You'll get the basics in understandable form.

Ratey, J. (2008) Neuroscience and the brain: Implications for counseling and therapy (Video). Framingham, MA: Microtraining Associates.

Here you will find the "guts" of the User's Guide (listed above) in a video presentation. 
Sapolsky, R. (2010) Biology and human behavior: The neurological origins of individuality, 2nd Ed. (Audio). Chantilly, VA: The Teaching Company. (www.teach12.com, http://en.wikipedia.org/wiki/Robert_Sapolsky)

Audio or DVD presentations by one of the masters of the field. Listen to or view this and you'll have a good solid beginning scientific understanding.

\section{Future Action Strategies}

Conference participants and presenters established the following action points.

Learn more about the importance of neuroscience in counseling

We believe that each counselor, committed to social justice, has the responsibility to start studying neuroscience and related fields. This will be time-consuming, but it necessary to be ready for the next decade.

Apply the information learned to myself and with others

Exercise, meditation, social relationships, and a general wellness orientation for the helper are becoming more essential. How effective are you at stress management. Moving beyond the personal, clients who experience social injustice need exactly the same thing that you do. The other key applications of this knowledge is increased awareness that oppression of all types, whether abuse, economic, racial, etc., all injure the individual and can cause serious injury over time.

\section{Educate colleagues and students}

Not only do we have a responsibility to learn ourselves, we also need to teach this information to others. Moreover, clients will profit from psychoeducation and this should help compliance and facilitate a more positive, wellness orientation.

Stress from social injustice negatively impacts wellness

Thus, advocate and ACT for Social Justice. Neuroscience clearly points out that environmental contingencies can harm human development.

Emphasize positive-not dwell on negatives

Wellness and positive societal change will make a difference.

Integrate more neuroscience education for clients, encourage stress reduction, and encourage exercise and wellness

Also in CACREP and Code of Ethics. Stress management is coming to be the major treatment of choice. Other therapies can be used to supplement this core. But stress management really needs to be reframed as positive living. 
Contact information/Correspondence:

Allen E. Ivey, Ed.D.

PO Box 707

Sunapee, $\mathrm{NH}$ 03782-0707

E-mail: allenivey@gmail.com

Allen E. Ivey, Ed.D., is Distinguished University Professor (Emeritus), University of Massachusetts, Amherst, and Professor of Counseling at the University of South Florida, Tampa (courtesy appointment); Carlos P. Zalaquett, Ph.D., is Associate Professor and Coordinator of the Clinical Mental Health Counseling Specialization in the Psychological and Social Foundations Department, University of South Florida, Tampa.

\section{References}

American Psychological Association (2000). Resolution on poverty and socioeconomic status. Retrieved from http://www.apa.org/pi/urban/povres.html.

Benson, H., \& Proctor, W. (2010). Relaxation revolution: Enhancing your personal health through the science and genetics of mind body healing. New York, N.Y.: Scribner.

Cacioppo, J. T., Berntson, G. G., \& Decety, J. (2010). Social neuroscience: and its relationship to social psychology. Social Cognition, 28, 675-685.

Decety, J., \& Jackson, P. (2004). The functional architecture of human empathy. Behavioral and Cognitive Neuroscience Reviews, 3(271-100). Retrieved from http://home.uchicago.edu/\%7Edecety/publications/Decety CDPS06.pdf.

Gazzaniga, M. S. (2008). Human: The science behind what makes us unique. New York: Harper Collins Publishers.

Grawe, K. (2007). Neuropsychotherapy: How the neurosciences inform effective psychotherapy. Mahway, NJ: Erlbaum.

Hackman, D.A., Farah, M.J. \& Meaney, M.J. (2010). Socioeconomic status and the brain: Mechanistic insights from human and animal research. Nature Reviews Neuroscience, 11, 651-659.

Ivey, A. (2009). Neuroscience and counseling: Implications for microskills and practice (Video). Framingham, MA: Microtraining Associates. (www.emicrotraining.com). 
Ivey A., Ivey, M., \& Zalaquett, C. (2010). Intentional interviewing and counseling: Facilitating client development in a multicultural world, $7^{\text {th }}$ ed. Belmont, CA: Brooks/Cole/Cengage.

Likhtik, E., Popa,D., Apergis-Schoute, J., Fidacaro, G. A., \& Paré, D. (2008). Amygdala intercalated neurons are required for expression of fear extinction. Nature, 454 , 642-645.

Ratey, J. (2001). A user's guide to the brain. New York, NY: Vintage.

Ratey, J. (2008). Neuroscience and the brain: Implications for counseling and therapy (Video). Framingham, MA: Microtraining Associates.

Sapolsky, R. (2010). Biology and human behavior: The neurological origins of individuality, 2nd Ed. (Audio). Chantilly, VA: The Teaching Company. (www.teach12.com, http://en.wikipedia.org/wiki/Robert Sapolsky).

Schwartz, J., \& Begley, S. (2003). The mind and the brain: Neuroplasticity and the power of mental force. New York: Regan. 